\title{
Inadaptación escolar en hijos de padres separados
}

\section{Eugenia Piñero Ruiz, Carmen Godoy Fernández, y Pilar Gandía Herrero Universidad de Murcia (España)}

Los procesos de separación y divorcio suponen una reestructuración de las relaciones, las formas de convivencia y las rutinas de todos los miembros de la familia. En ocasiones, estas rupturas suponen un conflicto, desarrollándose como procesos judiciales contenciosos. Se ha documentado la existencia de un mayor riesgo de inadaptación en los hijos de padres inmersos en procesos de separación o divorcio con un alto nivel de conflictividad, y a que experimenten dificultades en varios contextos, el personal, el social, y específicamente en el ámbito académico. Nuestro estudio, realizado sobre 246 hijos de padres en proceso de separación o divorcio, con edades comprendidas entre los 8 y los 18, mostró predominio de insatisfacción con la relación que los padres mantenían entre sí, además de mayores niveles de inadaptación escolar, sobre todo en el caso de los hijos varones y aumentando en las edades superiores. Se establece como conclusión que existen diferencias en la adaptación escolar de los menores varones cuyos padres están involucrados en un proceso de separación o divorcio de alta conflictividad. El sexo y la edad pueden considerarse factores relacionados, mostrándose las mujeres más adaptadas a nivel escolar, así como los hijos de menor edad.

Palabras clave: Inadaptación escolar, divorcio, familia, conflicto.

School maladjustment in children of divorced parents. Separation and divorce processes suppose a restructuration of family relationships, of the ways of convivence and the habits of all family members. Sometimes, these familly processes becomes in a conflictive situation that developes as a contentious judicial process. The existence of an increased risk of maladjustment in sons and daughters of couples in high conflictive separation or divorce processes have been documented. They frecuently experience more difficulties in some contexts, as personal, social and specifically in academic environment. Our investigation, conducted on 246 sons and daughters whose parents were in separation or divorce processes, aged between 8 and 18, showed a predominance of dissatisfaction with parents relationship, in addition to higher levels of school maladjustment, especially in the case of male and increasing in older boys. Our conclusions are that there are differences in school adjustment of students whose parents are involved in high conflict separation or divorce processes. Sex and age may be related factors, with girls better adapted, as well as younger boys.

Keywords: Academic adjustment, divorce, family, conflict.

Correspondencia Eugenia Piñero Ruiz. Facultad de Psicología. Universidad de Murcia. Campus de Espinardo. C.P.: 30100. Murcia (España). E-mail: eugeniapr@um.es 
Los procesos de separación y divorcio suponen una reestructuración de las relaciones, las formas de convivencia y las rutinas de todos los miembros de la familia. Generalmente, las rupturas de parejas con hijos suelen realizarse de mutuo acuerdo, logrando los progenitores soluciones que favorecen la adaptación de los menores. En ocasiones, estas rupturas suponen un conflicto en el que terceros han de intervenir para lograr acuerdos. Estos casos suelen dirimirse en los juzgados, estableciéndose sentencias en las que se toman decisiones acerca de las formas de convivencia y relación tras la ruptura de pareja.

De la propia ley nace la necesidad de justificar con criterios científicos y recurrir a un mayor número de recursos en los que apoyarse a la hora de tomar decisiones, entre los que la psicología juega un papel fundamental (Marín, Dujo, y Horcajo, 2017). En muchos de los procedimientos de divorcio contencioso, en los que se ha de decidir el modelo de relación que tendrán tras el divorcio padres e hijos, se cuenta con la intervención de expertos psicólogos forenses, que tras una evaluación psicológica del núcleo familiar, recomiendan las mejores alternativas de guarda y custodia y/o sistema de visitas para los sujetos evaluados (Arch, 2008; Rodríguez, Carbonell, y Jarne, 2014).

Aunque la mayoría de los descendientes de progenitores separados se adapta satisfactoriamente a esta nueva situación, se ha documentado la existencia de un mayor riesgo de inadaptación en los hijos de padres inmersos en procesos de separación o divorcio con un alto nivel de conflictividad, y una mayor frecuencia de dificultades en varios contextos, el personal, el social, y específicamente en el ámbito académico (Fariña y Arce, 2008). Este tipo de procesos de separación se caracterizan por reiterados desacuerdos, dificultades de comunicación, posicionamiento de los menores y/o excesiva judicialización de las relaciones familiares. En línea con lo expuesto, varios estudios han hallado diferencias en los niveles de malestar emocional (Gómez-Ortiz, Martín, y Ortega-Ruiz, 2017) y en la inadaptación escolar de los menores en situaciones de conflicto familiar en comparación con la población general (Sanchís, Robredo, Llop, y Carbonell, 2017). A través del estudio de las recomendaciones de custodia por parte de peritos tras el examen psicológico-forense familiar, se ha encontrado que cuando el menor se enfrenta a una mala adaptación escolar tras la separación de sus progenitores, la proporción de recomendaciones hacia otras personas distintas al padre y la madre, así como la institucionalización, es significativamente superior (Gandía, 2016). Sin embargo, en este estudio, otras áreas exploradas, como psicopatología, presencia de conductas disruptivas o apoyo social percibido en los menores, no han supuesto una relación significativa con la decisión pericial.

Investigaciones en nuestro país han detectado peores consecuencias en los hijos varones, sobre todo en los dos años siguientes a la separación (Cantón y Justicia, 2002) y diferencias en función de la edad, aunque con datos contradictorios. 
Con este estudio se pretendió conocer el grado de inadaptación escolar que muestran niños y niñas y adolescentes descendientes de progenitores en procesos de separación y/o divorcio contencioso, así como buscar posibles diferencias en relación al sexo y la edad de los menores.

\section{MÉTODO}

\section{Participantes}

La muestra estuvo integrada por 246 hijos de padres en proceso de separación o divorcio (117 varones y 129 mujeres), con edades comprendidas entre los 8 y los 18 años de la Región de Murcia.

\section{Instrumento}

Se aplicó de forma individual el Test Adaptativo Multifactorial de Adaptación Infantil (TAMAI) de P. Hernández aplicable a sujetos de edades comprendidas entre los 8 años y la edad adulta. El instrumento permite evaluar, a través de 175 preguntas, en base a las respuestas del sujeto, los niveles de inadaptación personal, social, escolar y familiar. Su aplicación se divide en tres niveles que se corresponden con los siguientes cursos académicos: el Nivel I a $3^{\circ}, 4^{\circ}$ y $5^{\circ}$ de primaria, el Nivel II a $6^{\circ}$ de primaria y $1^{\circ}$ y $2^{\circ}$ de Educación Secundaria Obligatoria y Nivel III, $3^{\circ}$ y $4^{\circ}$ de Educación Secundaria Obligatoria y $1^{\circ}$ y $2^{\circ}$ de Bachiller, además de adultos. Las diferentes escalas de Adaptación general (suma de las puntuaciones en la personal, social, escolar y familiar) personal (desajuste que presenta la persona en relación a ella misma y a su realidad), social (problemas o incapacidad en las relaciones sociales), escolar (insatisfacción y comportamiento inadecuado en la realidad escolar) y familiar (insatisfacción con la situación familiar y/o con los hermanos), están formadas por subescalas que reflejan aspectos diferentes dependiendo del nivel de aplicación. En concreto, la escala de Inadaptación escolar, en la que se centra este trabajo, engloba las puntuaciones en Inadaptación escolar externa, Aversión a la instrucción y Aversión al aprendizaje para el Nivel I, Aversión a la instrucción, Hipolaboriosidad, Hipomotivación, Aversión al profesorado e Indisciplina para el Nivel II y Aversión a la instrucción, Hipolaboriosidad, Hipomotivación, Insatisfacción escolar, Aversión al profesorado e Indisciplina en el Nivel III.

\section{Procedimiento}

Los participantes en el estudio fueron los hijos e hijas de progenitores separados inmersos en procesos contenciosos, en los que se dirimía principalmente el sistema de guarda y custodia y el sistema de visitas. Los datos de la investigación se obtuvieron durante las evaluaciones para la realización de informes psicológicos 
periciales en estos procedimientos, extrayéndose los datos del estudio a partir de la evaluación psicológica pericial.

Se obtuvo el consentimiento informado de todas las familias evaluadas, que concedieron su permiso expreso para el uso de los datos de la evaluación para fines de investigación. Se compararon los niveles de inadaptación comparando a varones y mujeres, además de valorando su evolución en los distintos niveles. También se comparó a los que presentaban alta insatisfacción con el ambiente familiar en las puntuaciones de inadaptación escolar, con los que se mostraban satisfechos con el clima familiar después de haber descrito los niveles de satisfacción de la muestra.

\section{RESULTADOS}

En primer lugar, se presentan los resultados obtenidos en todos los niveles de inadaptación que evalúa el instrumento empleado (TAMAI). Como podemos comprobar en la tabla 1, se incluyen las puntuaciones en Inadaptación general, personal, social y escolar. Se encuentran diferencias significativas en el caso de la Inadaptación escolar, encontrándose una mayor puntuación en inadaptación al ámbito escolar en los varones del Nivel I, que se encuentran escolarizados entre el tercer y quinto curso de la educación primaria.

Tabla 1. Inadaptación en varones y mujeres del Nivel I

\begin{tabular}{lcccc}
\hline \multicolumn{3}{c}{ NIVEL I } \\
\hline & $\begin{array}{c}\text { VARONES } \\
(n=56)\end{array}$ & \multicolumn{2}{c}{$\begin{array}{c}\text { MUJERES } \\
(n=69)\end{array}$} \\
\hline & $m$ & $D T$ & $m$ & $D T$ \\
\hline Inadaptación General & 23.92 & 10.86 & 21.34 & 12.44 \\
\hline Inadaptación Personal & 8.46 & 4.28 & 8.98 & 5.71 \\
\hline Inadaptación Social & 8.28 & 4.53 & 7.30 & 4.59 \\
\hline Inadaptación Escolar & $7.19 *$ & 5.1 & 5.05 & 4.79 \\
\hline Nota: *significativo a nivel .05. & &
\end{tabular}

En cuanto a las puntuaciones en los diferentes tipos de Inadaptación en el Nivel II (Tabla 2), que corresponde a los menores de sexto curso de educación primaria y primero y segundo de educación secundaria obligatoria, encontramos una tendencia similar a la descrita en el Nivel I, con diferencias significativas a nivel estadístico en la Inadaptación escolar, que es mayor en los varones. 
Tabla 2. Inadaptación en varones y mujeres del Nivel II

\begin{tabular}{lcccc}
\hline \multicolumn{4}{c}{ NIVEL II } \\
\hline & $\begin{array}{c}\text { VARONES } \\
(n=47)\end{array}$ & \multicolumn{2}{c}{$\begin{array}{c}\text { MUJERES } \\
(n=37)\end{array}$} \\
\hline & $m$ & $D T$ & $m$ & $D T$ \\
\hline Inadaptación General & 25.83 & 13.63 & 23.27 & 13.03 \\
\hline Inadaptación Personal & 6.38 & 4.81 & 7.40 & 5.68 \\
\hline Inadaptación Social & 9.34 & 5.21 & 8.27 & 4.14 \\
\hline Inadaptación Escolar & $10.10 *$ & 7.07 & 7.59 & 5.45 \\
\hline Nota: *significativo a nivel de .05. & & &
\end{tabular}

Por último, en el Nivel III (Tabla 3), correspondiente a menores de tercero y cuarto de educación secundaria obligatoria, así como primero y segundo de bachiller, observamos de nuevo una puntuación significativamente superior en la Inadaptación escolar de los varones, al compararse con la Inadaptación escolar que presentan las mujeres. En cuanto al nivel de Inadaptación personal entre ambos grupos, se encontraron diferencias significativas entre varones y mujeres, siendo mayor en las primeras que en los segundos.

Tabla 3. Inadaptación en varones y mujeres del Nivel III

\begin{tabular}{lcccc}
\hline \multicolumn{5}{c}{ NIVEL III } \\
\hline & $\begin{array}{c}\text { VARONES } \\
(n=13)\end{array}$ & \multicolumn{2}{c}{$\begin{array}{c}\text { MUJERES } \\
(n=23)\end{array}$} \\
\hline & $m$ & $D T$ & $m$ & $D T$ \\
\hline Inadaptación General & 34.61 & 15.14 & 32.47 & 13.63 \\
\hline Inadaptación Personal & 9.61 & 4.97 & $13.65^{*}$ & 6.62 \\
\hline Inadaptación Social & 11.76 & 6.35 & 9.78 & 5.08 \\
\hline Inadaptación Escolar & $13.23^{*}$ & 7.00 & 9.04 & 6.24 \\
\hline Nota: *significativo a nivel de .05. & & &
\end{tabular}

El segundo bloque de resultados corresponde a las comparaciones en las puntuaciones de varones y mujeres en cuanto a los factores específicos que componen la puntuación en Inadaptación escolar (Inadaptación escolar externa, Aversión a la instrucción y Aversión al aprendizaje) para cada uno de los tres niveles de evaluación.

Como podemos comprobar en la tabla 4, todas las puntuaciones son superiores en varones al compararlas con las de las mujeres, encontrando además que estas diferencias son significativas en las escalas de Inadaptación escolar externa y Aversión al aprendizaje. 
PIÑERO et al. Inadaptación escolar en hijos de padres separados

Tabla 4. Inadaptación escolar en varones y mujeres del Nivel I

\begin{tabular}{lcccc}
\hline \multicolumn{3}{c}{ NIVEL I } \\
\hline & $\begin{array}{c}\text { VARONES } \\
(n=56)\end{array}$ & $\begin{array}{c}\text { MUJERES } \\
(n=69)\end{array}$ \\
\hline Inadaptación escolar externa & $2.02^{*}$ & 2.33 & 0.94 & 1.53 \\
\hline Aversión a la instrucción & 9.66 & 4.84 & 6.92 & 4.37 \\
\hline Aversión al aprendizaje & $3.36^{*}$ & 2.93 & 2.03 & 2.40 \\
\hline Nota: *significativo a nivel de .05. & & & &
\end{tabular}

En el Nivel II (Tabla 5) observamos puntuaciones superiores en los varones en todas las escalas de la Inadaptación escolar (Aversión a la instrucción, Hipolaboriosidad, Hipomotivación, Aversión al profesorado e Indisciplina), siendo significativas las diferencias en Aversión a la instrucción e Hipolaboriosidad.

\begin{tabular}{|c|c|c|c|c|}
\hline \multicolumn{5}{|c|}{ NIVEL II } \\
\hline & \multicolumn{2}{|c|}{$\begin{array}{c}\text { VARONES } \\
(n=46)\end{array}$} & \multicolumn{2}{|c|}{$\begin{array}{c}\text { MUJERES } \\
(n=36)\end{array}$} \\
\hline & $m$ & $D T$ & $m$ & $D T$ \\
\hline Aversión a la instrucción & $9.06^{*}$ & 5.99 & 7.00 & 4.79 \\
\hline Hipolaboriosidad & $2.69 *$ & 2.26 & 1.69 & 1.92 \\
\hline Hipomotivación & 4.95 & 2.95 & 4.38 & 2.80 \\
\hline Aversión al profesorado & 1.41 & 1.54 & 0.91 & 1.29 \\
\hline Indisciplina & 1.26 & 1.56 & 0.77 & 1.17 \\
\hline
\end{tabular}

Por último, en la tabla 6, correspondiente al Nivel III, podemos comprobar que de nuevo son superiores las puntuaciones en todas las escalas en los varones, hallando diferencias significativas a nivel estadístico en las escalas de Hipolaboriosidad e Indisciplina.

Tabla 6. Inadaptación escolar en varones y mujeres del Nivel III

\begin{tabular}{lcccc}
\hline \multicolumn{3}{c}{ NIVEL III } \\
\hline & $\begin{array}{c}\text { VARONES } \\
(n=13)\end{array}$ & \multicolumn{2}{c}{$\begin{array}{c}\text { MUJERES } \\
(n=23)\end{array}$} \\
\hline Aversión a la instrucción & 11.84 & 5.66 & 8.73 & 5.88 \\
\hline Hipolaboriosidad & $5.30^{*}$ & 2.62 & 3.82 & 2.38 \\
\hline Hipomotivación & 4.69 & 2.59 & 3.47 & 2.85 \\
\hline Aversión al profesorado & 1.46 & 1.50 & 1.00 & 1.62 \\
\hline Indisciplina & $1.38^{*}$ & 1.75 & 0.30 & 0.76 \\
\hline Nota: *significativo a nivel de .05. & & &
\end{tabular}


En cuanto a la evolución de la Inadaptación escolar en función del nivel en el que se encuentran los menores, en base a las clasificaciones propuestas por el cuestionario ( $\mathrm{MB}=$ Muy baja; $\mathrm{B}=$ Baja; $\mathrm{CB}$ : Casi baja; $\mathrm{M}=$ Media; $\mathrm{CA}=\mathrm{Casi}$ alta; $\mathrm{A}=$ Alta y $\mathrm{MA}=$ Muy alta), podemos comprobar en la Tabla 5 que no existen diferencias entre los diferentes niveles valorados a través de sus puntuaciones medias. Sin embargo, sí se observa que la adaptación académica es ligeramente superior en las mujeres de los niveles II y III.

\begin{tabular}{|c|c|c|c|}
\hline \multicolumn{4}{|c|}{ NIVEL I } \\
\hline & $m$ & $D T$ & Clasificación TAMAI \\
\hline Varones & 7.19 & 5.10 & $\mathrm{M}$ \\
\hline Mujeres & 5.05 & 4.79 & $\mathrm{M}$ \\
\hline \multicolumn{4}{|c|}{ NIVEL II } \\
\hline Varones & 10.10 & 7.07 & M \\
\hline Mujeres & 7.59 & 5.45 & $\mathrm{CB}$ \\
\hline \multicolumn{4}{|c|}{ NIVEL III } \\
\hline Varones & 13.23 & 7.00 & $\mathrm{M}$ \\
\hline Mujeres & 9.04 & 6.24 & $\mathrm{CB}$ \\
\hline
\end{tabular}

En cuanto a la distribución de la muestra siguiendo la clasificación del cuestionario TAMAI, en la tabla 8 podemos comprobar, en el caso de los varones, que parece irse produciendo un aumento en los casos clasificados como Casi altos, Altos y Muy altos, al ir aumentando la edad de los sujetos. El porcentaje de clasificados en la escala Muy bajo oscila, con un sujeto en el Nivel III, igual que en la clasificación Bajo. La clasificación en Casi Bajo parece mantenerse estable en todos los niveles.

En cuanto a las mujeres, se observan tendencias similares en los tres niveles, excepto en la clasificación Muy bajo, que parece aumentar en el Nivel III con respecto al II, aunque no sí se compara con el I, y la clasificación en Muy alto, que aumenta en los Niveles II y III en comparación con el Nivel I.

Tabla 8. Clasificación de la Inadaptación escolar en varones y mujeres según Nivel

\begin{tabular}{lcccccccccccccc}
\hline & \multicolumn{1}{c}{} & \multicolumn{1}{c}{ MB } & \multicolumn{1}{c}{ B } & \multicolumn{1}{c}{ CB } & \multicolumn{1}{c}{ M } & \multicolumn{1}{c}{ CA } & A & \multicolumn{2}{c}{ MA } \\
\hline & $N$ & $\%$ & $n$ & $\%$ & $N$ & $\%$ & $n$ & $\%$ & $n$ & $\%$ & $n$ & $\%$ & $n$ & $\%$ \\
\hline Varones & 4 & 7.1 & 10 & 17.9 & 12 & 21.4 & 17 & 30.4 & 10 & 17.9 & 2 & 3.6 & 1 & 1.8 \\
\hline Mujeres & 8 & 11.6 & 16 & 23.2 & 14 & 20.3 & 18 & 26.1 & 7 & 10.1 & 4 & 5.8 & 2 & 2.9 \\
\hline & & & & \multicolumn{1}{c}{ NIVEL 2 } & & & & & & & \\
\hline Varones & 8 & 11.6 & 16 & 23.2 & 14 & 20.3 & 18 & 26.1 & 7 & 10.1 & 4 & 5.8 & 2 & 2.9 \\
\hline Mujeres & 1 & 2.7 & 12 & 32.4 & 7 & 18.9 & 5 & 13.5 & 8 & 21.6 & 3 & 8.1 & 0 & 0 \\
\hline & & & & \multicolumn{1}{c}{ NIVEL 3 } & & & & & & \\
\hline Varones & 1 & 7.7 & 1 & 7.7 & 3 & 23.1 & 5 & 38.5 & 1 & 7.7 & 1 & 7.7 & 1 & 7.7 \\
\hline Mujeres & 4 & 17.4 & 6 & 26.1 & 4 & 17.4 & 2 & 8.7 & 5 & 21.7 & 1 & 4.3 & 1 & 4.3 \\
\hline
\end{tabular}


Por último, se trató de comprobar si existían diferencias en la Inadaptación escolar de los menores en función de su Insatisfacción con el ambiente familiar.

Como se puede comprobar en la tabla 9, en todos los niveles y tanto en varones como en mujeres, el número de menores que indicaban algún grado de Insatisfacción con el ambiente familiar supera a los que no indicaban ningún grado de este tipo de insatisfacción. En cuanto a las diferencias en la puntuación en Inadaptación escolar dependiendo de la existencia o no de Insatisfacción familiar, no se encuentran diferencias significativas, aunque se puede observar una tendencia a que las puntuaciones sean superiores, excepto en las mujeres de los Niveles I y III.

Tabla 9. Inadaptación escolar en varones y mujeres según su Insatisfacción con el ambiente familiar NIVEL I VARONES

\begin{tabular}{|c|c|c|c|}
\hline \multicolumn{4}{|c|}{ NIVEL I VARONES } \\
\hline & \multicolumn{3}{|c|}{$\begin{array}{l}\text { Insatisfacción ambiente familiar No insatisfacción ambiente familiar } \\
\qquad(n=28)\end{array}$} \\
\hline & $\overline{D T}$ & $m$ & $\overline{D T}$ \\
\hline Inadaptación escolar & 7.75 & 6.90 & 5.70 \\
\hline \multicolumn{4}{|c|}{ NIVEL I MUJERES } \\
\hline & \multicolumn{3}{|c|}{$\begin{array}{l}\text { Insatisfacción ambiente familiar No insatisfacción ambiente familiar } \\
\qquad(n=24)\end{array}$} \\
\hline Inadaptación escolar & 5.21 & 5.92 & 4.06 \\
\hline \multicolumn{4}{|c|}{ NIVEL II VARONES } \\
\hline & \multicolumn{3}{|c|}{$\begin{array}{l}\text { Insatisfacción ambiente familiar No insatisfacción ambiente familiar } \\
\qquad(n=32)\end{array}$} \\
\hline Inadaptación escolar & 9.90 & 9.20 & 7.69 \\
\hline \multicolumn{4}{|c|}{ NIVEL II MUJERES } \\
\hline & \multicolumn{3}{|c|}{$\begin{array}{l}\text { Insatisfacción ambiente familiar No insatisfacción ambiente familiar } \\
\qquad(n=26)\end{array}$} \\
\hline Insatisfacción escolar & 7.84 & 6.50 & 3.61 \\
\hline \multicolumn{4}{|c|}{ NIVEL III VARONES } \\
\hline & \multicolumn{3}{|c|}{$\begin{array}{l}\text { Insatisfacción ambiente familiar No insatisfacción ambiente familiar } \\
\qquad(n=9)\end{array}$} \\
\hline Insatisfacción escolar & 13.22 & 10.00 & 2.82 \\
\hline \multicolumn{4}{|c|}{ NIVEL III MUJERES } \\
\hline & \multicolumn{3}{|c|}{$\begin{array}{l}\text { Insatisfacción ambiente familiar No insatisfacción ambiente familiar } \\
\qquad(n=18)\end{array}$} \\
\hline Insatisfacción escolar & 7.66 & 12.00 & 5.29 \\
\hline
\end{tabular}




\section{DISCUSIÓN Y CONCLUSIONES}

Aunque en nuestro estudio no hemos valorado las posibles diferencias entre los datos de nuestra muestra y los de población general en cuanto a niveles de Inadaptación, diversos estudios han subrayado que los hijos de padres divorciados presentan menos calidad de vida relacionada con distintos parámetros, como pueden ser la salud, el bienestar y el rendimiento académico (Orgilés y Samper, 2011).

En este sentido, los resultados del trabajo reflejan que el número de menores que indicó Insatisfacción con el ambiente familiar superó a los que no lo presentaban. En el caso de los varones, encontramos un $79.3 \%$ que manifestó insatisfacción con la relación que los padres mantenían entre sí frente al $20.6 \%$ que no refirió dicha insatisfacción. Y en el caso de las mujeres, un $74.7 \%$ frente al $25.2 \%$ que no indicó insatisfacción con el clima relacional y la relación que los padres mantenían después del divorcio.

En cuanto a la adaptación global, otros estudios que han empleado el mismo instrumento utilizado en esta investigación, han hallado que el proceso de separación repercute en los hijos, encontrándose mayores niveles de inadaptación personal, familiar, escolar y social (Fariña y Arce, 2008).

Los varones obtienen mayores puntuaciones en inadaptación escolar que las mujeres, pero las mujeres superan a los varones en inadaptación personal en edades superiores. Otras investigaciones no han encontrado diferencias significativas del efecto del divorcio en el rendimiento según sexo (Amato, 2001; Sun y Li, 2001). Todas las puntuaciones que cargan en Inadaptación escolar son superiores en varones al compararlas con las mujeres, coincidiendo las diferencias más significativas en la Hipolaboriosidad y destacando en el grupo de adolescentes entre catorce y diecisiete años los problemas de indisciplina.

Algunas investigaciones evidencian que los efectos del divorcio sobre el rendimiento educativo son más negativos cuando el divorcio sucede en etapas más tempranas (Jeynes, 2002) y otras investigaciones, sin embargo encuentran que el efecto es mayor en la adolescencia (Anthony, DiPerna, y Amato, 2014). Acorde a los resultados de este trabajo, las repercusiones en la inadaptación para los varones se mantienen en edades superiores, es decir, son más estables las dificultades a lo largo del ciclo académico y en el caso de las mujeres se evidencia que la adaptación escolar es ligeramente superior entre los once y diecisiete años. Estos datos pueden ser concordantes con los obtenidos por Magnuson y Berger (2012) que afirmaban que las transiciones familiares se asociaban a cambios negativos en comportamiento y logro académico entre los seis y doce años de edad. En esta muestra parece cumplirse dicha afirmación para el caso de las mujeres. 
En relación a la existencia o no de mayores niveles de Inadaptación escolar en función de la Insatisfacción con el ambiente familiar, se ha podido observar una tendencia a ser superiores en los varones, como han indicado estudios que subrayan la mayor probabilidad de fracaso escolar en hijos de padres divorciados en situación de conflicto frente a familias biparentales o divorciados sin conflicto (Escapa, 2017; Morán, 2012; Valdés, Martínez, Urías, e Ibarra, 2011).

Podríamos concluir, según los datos arrojados, que existen diferencias estadísticamente significativas en la adaptación escolar de los menores varones cuyos padres están involucrados en un proceso de separación o divorcio de alta conflictividad. El sexo y la edad pueden considerarse factores relacionados, mostrándose las mujeres más adaptadas a nivel escolar, así como los hijos de menor edad.

Se plantean, como aspectos de mejora para el trabajo, la posibilidad de realizar comparaciones de la muestra de hijos e hijas de progenitores en procesos de separación y/o divorcio, con muestras procedentes de población general y con hijos de padres separados que hayan logrado procesos de adaptación sin conflictividad significativa.

\section{REFERENCIAS}

Amato, P. (2001). Children of divorce in the 1990s: An update of the Amato and Keit in 1991 (meta-analysis). Journal of Family Psychology, 15(3), 355-370.

Anthony, C.J., DiPerna, J.C., y Amato, P.R. (2014). Divorce, approaches to learning, and children's academic achievement: A longitudinal analysis of mediated and moderated effects. Journal of School Psychology, 52(3), 249-261.

Arch, M. (2008). La intervención de los psicólogos forenses en las evaluaciones de la guarda y custodia de los niños (tesis doctoral). Barcelona: Universidad de Barcelona.

Cantón, J. y Justicia, M.D. (2002). Características del niño y adaptación al divorcio de los padres. En J. Cantón, M.R. Cortés y M.D. Justicia. (Eds), Conflictos matrimoniales, divorcio y desarrollo de los hijos (pp.47-66). Madrid: Ediciones Pirámide.

Escapa, S. (2017). Los efectos del conflicto parental después del divorcio sobre el rendimiento educativo de los hijos. Revista Española de Investigaciones Sociológicas, 158, 41-58. doi:10.5477/cis/reis. 158.41

Fariña, F., y Arce, R. (2008). Intervención psicosocial con progenitores en ruptura de pareja. En J.A. González y J.C. Núñez. (Eds), Psicología y educación: Un lugar de encuentro (pp. 2423-2434). Oviedo: Ediciones de la Universidad de Oviedo.

Gandía, P. (2016). Informes de recomendación de guarda y custodia en procesos de ruptura de pareja: variables psicológicas y sociofamiliares (Tesis doctoral no publicada). Murcia: Universidad de Murcia.

Gómez-Ortiz, O., Martín, L., y Ortega-Ruiz, R. (2017). Conflictividad parental, divorcio y ansiedad infantil. Pensamiento Psicológico, 15(2), 67-78. doi:10.11144/Javerianacali.PPSI15-2.cpda

Jeynes, W.H. (2002). Does Parental Involvement Eliminate the Effects of Parental Divorce on the Academic Achievement of Adolescents? Journal of Divorce y Remarriage, 37(1), 101115. 
Magnuson, K., y Berger L.M. (2009). Family Structure States and Transitions: Associations with Children's Wellbeing During Middle Childhood. Journal Marriage and Family, 71(3), 575-591. doi:10.1111/j.1741-3737.2009.00620.x

Marín, M., Dujo, V., y Horcajo, P.J. (2017). Estudio comparativo de las decisiones de los magistrados del Tribunal Supremo español y los resultados de estudios empíricos sobre las implicaciones psicológicas en menores en situación de guarda y custodia compartida. Anuario de Psicología Jurídica, 27(1), 115-125. doi:10.1016/j.apj.2016.11.002

Morán, E. (2012). Factores de la actitud y ansiedad al aprendizaje de la matemática en estudiantes adolescentes de la ciudad de Milagro. La relación de la estructura familiar y el rendimiento académico. Unión Revista Iberoamericana de Educación Matemática, 29, 109-120.

Orgilés, M., y Samper, M.D. (2011). El impacto del divorcio en la calidad de vida de los niños de 8 a 12 años de edad en la provincia de Alicante. Gaceta Sanitaria, 25(6), 490-494.

Rodríguez, C., Carbonell, X., y Jarne, A. (2014). Revisión conceptual del peritaje psicológico en relación a las custodias de menores en Cataluña. Anuario de Psicología Jurídica, 24(1), 19-29.

Sanchís, A., Robredo, C., Llop, R., y Carbonell, E.J. (2017). Efectos del conflicto parental postdivorcio en la adaptación y bienestar de los hijos. Información Psicológica, 114, 8397.

Sun, Y., y Li, Y. (2009). Parental divorce, sibship size, family resources, and children's academic performance. Social Science Research, 38(3), 622-634.

Valdés, A.A., Martínez, A.C., Urías, M., e Ibarra, B.G. (2011) efectos del d divorcio de los padres en el desempeño académico y la conducta de los hijos. Enseñanza e Investigación en Psicología, 16(2), 295-308. 